\title{
Proceedings of the 7th international symposium on interventional electrophysiology
}

Published online: 16 August 2014

(C) Springer Science+Business Media New York 2014

Munich, September 2013

We are pleased to introduce this special issue of the Journal of Interventional Cardiac Electrophysiology that includes selected presentations from the International Symposium on Interventional Electrophysiology held in Munich on September 18-20, 2013. The seventh edition of this meeting continues the original concepts underlying this series of international symposia which had three major goals or "missions": Firstly, the meeting seeks to build bridges from basic science and pathophysiology in arrhythmology to applied science and clinical medicine. Secondly, to discuss the latest and most advanced technologies used in the treatment of arrhythmias, and thirdly, to debate the conceptual basis and results of clinical trials. This issue of the Journal of Interventional Electrophysiology presents a selection of lectures held at the Munich meeting which was subsequently translated into a full review of the subject:

A heavily discussed and yet unresolved area in catheter ablation of atrial fibrillation (AF) is the question about the most effective way to treat the substrate contributing to atrial arrhythmias. Dr. Narayan and colleagues present their method and concept of mapping and ablating stable sources of atrial fibrillation using the "FIRM" approach and hereby challenge the current "pulmonary vein oriented" concepts in AF ablation. In contrast to this debate, ablation of VF is currently exclusively targeting the initiating triggers: Dr. Willems and coworkers present us an excellent overview about identification and ablation of the initiating arrhythmias in various conditions like Brugada- or Long QT syndrome.

Identifying the target for ablation is one step, getting catheters to this spot in a safe and rapid manner is the major challenge for the operator. Ablating more complex arrhythmia substrates is generating longer procedure durations and significant fluoroscopy times. The latest status of the nonfluoroscopic catheter visualisation technique "MediGuide" is presented and discussed in two up-to-date manuscripts in this issue.

Stroke and embolism are the most important morbidity induced by AF. The Novel or Direct Oral Anticoagulants extend our treatment options significantly. We want to offer their benefits such as reduction of intracranial bleeding to our patients, but we also have to accept and solve new problems such as bleeding management in these patients without having an approved antidote. Drs. Ezekowitz and Levy give a wellbalanced overview about the clinical value of these new drugs and also summarize the current status of antidote development.

At the present time, cardiac pacing remains a cornerstone in arrhythmia and heart failure treatment. Drs. Nagarakanti and Saksena report a unique long-term experience in treating atrial fibrillation in an otherwise resistant patient group using a dual site atrial pacing concept, whereas Drs. Sethi and coworkers discuss practical issues in multisite pacing for heart failure treatment.

We hope the Journal readership and health care professionals interested in the latest directions in cardiac arrhythmia therapy and management will enjoy and increase his knowledge by reading this special issue and that we will finally meet our major challenge as physicians and researchers: to deliver optimal treatment to our patients.

Thorsten Lewalter

Sanjeev Saksena 